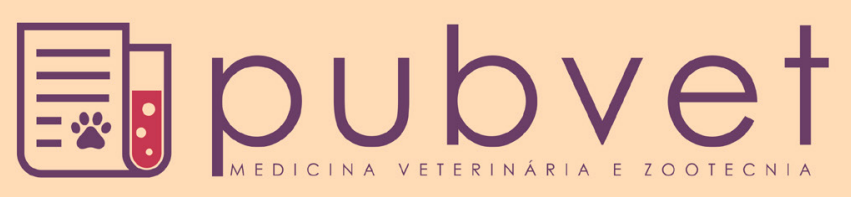

HTTP://DX.DOI.ORG/10.22256/PUBVET.V11N4.313-319

\title{
Viabilidade econômica de um produto a base de algas da associação de maricultoras do litoral Norte Potiguar
}

\author{
Josué dos Santos Júnior'1, Lucas de Oliveira Soares Rebouças²*, Julianna Paula do Vale \\ Figueiredo ${ }^{1}$, Ambrósio Paula Bessa Júnior ${ }^{3}$, Ivanilson de Souza Maia ${ }^{3}$
}

${ }^{I}$ Engenheiro(a) de Pesca, Mestrando do Programa de Pós-Graduação em Ciências Animais, Universidade Federal Rural do Semiárido (Mossoró, Rio Grande do Norte, Brasil-e-mail: josuejunior-07@hotmail.com; juliannafigueiredo@hotmail.com ${ }^{2}$ Engenheiro(a) de Pesca, Doutorando do Programa de Pós-Graduação em Ciências Animais, Universidade Federal Rural do Semiárido (Mossoró, Rio Grande do Norte, Brasil-e-mail: lucaslosr@gmail.com

${ }^{3}$ Professor do curso de Engenharia de Pesca da Universidade Federal Rural do Semiárido (Mossoró, Rio Grande do Norte, Brasil-e-mail: bessa@ufersa.edu.br; ivanilson.maia@ufersa.edu.br

*Autor para correspondência

\begin{abstract}
RESUMO. O objetivo do presente estudo foi identificar a sustentabilidade econômica do grupo das maricultoras do município Rio do fogo/RN - AMAR, com o intuito de identificar os dados econômicos provenientes da venda dos produtos desenvolvidos na comunidade e realizar o estudo de viabilidade econômico de um produto a base de alga. Para alcançar os dados coletados, foram realizadas oficinas e uma visita às áreas de cultivo. Como estratégia metodológica, utilizou-se o Diagnóstico Rural Participativo e suas ferramentas próprias de mobilização do conhecimento, que permitem reconstruir todos os passos processuais da atividade, sua participação no cotidiano da comunidade e da família, as relações sociais e econômicas, a gestão democrática e a viabilidade econômica desse empreendimento popular, no passado e no presente. A associação conta com 6 associadas ativas, produzindo sete produtos, o pó de algas, a alga seca in natura, sabonete, cocada, mousse, gelatina e biscoito. O pó de algas é responsável por $50 \%$ da renda da associação que é R\$1.800,00 totalizando em R \$300,00/associado. Para o estudo de viabilidade economia o produto escolhido foi o pó de algas. O estudo de viabilidade econômica demonstrou que é viável a produção do pó ou talco de algas, pois a venda deste produto contribuirá para aumento da renda das mulheres associadas. Renda esta que apesentou um crescimento de 43,3\% passando de uma média de $\mathrm{R} \$ 300,00$ para $\mathrm{R} \$ 530,00$ /mensal. A renda média per capita familiar das maricultoras passou de $\mathrm{R} \$ 342,00$ para $\mathrm{R} \$ 488,80$ após o estudo, ou seja, um crescimento de $29,9 \%$ cima da renda per capita familiar atual. Desta forma, os dados desta pesquisa apontam que a atividade econômica de venda de produtos originados a partir da alga G. birdiae desenvolvido pela Associação de Maricultoras de Rio do Fogo - AMAR possui grande relevância social e econômica para as famílias das maricultoras da comunidade de Rio do Fogo.
\end{abstract}

Palavras chave: Comunidades costeiras, glacilaria birdiae, maricultura, sustentabilidade

\section{Economic feasibility study of a base product of algae in an association of maricultores of the North coast Potiguar}

\begin{abstract}
The objective of the present study was to identify the economic sustainability of the group of the maricultores of the municipality of Rio do Fogo / RN AMAR, in order to identify the economic data from the sale of products developed in the community and carry out the economic viability study of a product The base of kelp. To reach the data collected, workshops and a visit to the cultivated areas were carried out. As a methodological strategy, Participatory Rural Diagnosis and its own tools for knowledge
\end{abstract}


mobilization were used to reconstruct all the procedural steps of the activity, its participation in the daily life of the community and the family, social and economic relations, democratic and The economic viability of this popular enterprise, past and present. The association has 6 active associates, producing seven products, algae powder, fresh seaweed, soap, coconut, mouse, gelatin and biscuit. The algae powder is responsible for $50 \%$ of the income of the association that is $\mathrm{R} \$ 1,800.00$ totaling $\mathrm{R} \$ 300.00$ / associate. For the economic feasibility study the product chosen was algae powder. The economic feasibility study has shown that the production of algae powder or talc is feasible, since the sale of this product will contribute to increase the income of associated women. This income rose by $43.3 \%$ from an average of $\mathrm{R} \$ 300.00$ to $\mathrm{R} \$ 530.00 /$ month. The average family per capita income of the maricultoras increased from R \$ 342.00 to R $\$ 488.80$ after the study, or a $29.9 \%$ increase in the current per capita family income. In this way, the data of this research indicate that the economic activity of sale of products originated from the G. birdiae algae developed by the Association of Maricultoras of Rio do Fogo - AMAR has great social and economic relevance for the families of the maricultoras of the community of Rio do Fogo.

Keywords: Mariculture, Gracilaria birdiae, sustainability, coastal communities

\section{Viabilidad económica de un producto a base de algas de la asociación de maricultoras de la Costa Norte Potiguar}

RESUMEN. El objetivo de este estudio fue identificar la sostenibilidad económica del grupo de maricultoras del municipio Río de Fogo / RN - AMAR, con el fin de identificar los datos económicos provenientes de la venta de productos originados en la comunidad y llevar a cabo el estudio sobre la viabilidad económica de un producto a base de algas. Para conseguir los datos colectados, hubo talleres y una visita a las zonas de cultivo. Como estrategia metodológica se utilizó el Diagnostico Rural Participativo y sus propias herramientas de movilización de conocimiento, que permiten reconstruir todas las etapas del procedimiento de la actividad, su participación en la vida diaria de la comunidad y la familia, las relaciones sociales y económicas, la gestión democrática, y la viabilidad económica de este desarrollo popular, en pasado y presente. La asociación cuenta con seis afiliadas activas, promoviendo siete productos, el polvo de algas, algas secas fresca, jabón, cocadas, mousse, gelatina y galletas. El polvo de algas es responsable por el $50 \%$ de los ingresos de la asociación correspondiendo al valor de $\mathrm{R} \$ 1.800,00$ totalizando $\mathrm{R} \$ 300.00$ / asociado. Para el estudio de viabilidad económica el producto elegido fue el polvo de algas. El estudio de viabilidad económica demostró que es factible la producción de polvo o talco de algas, pues la venta de este producto contribuirá para aumentar los ingresos de las mujeres asociadas. Esta renta presentó un incremento de ingresos de 43,3\%, pasando en promedio de $\mathrm{R} \$ 300,00$ para $\mathrm{R} \$ 530.00$ / mes. El ingreso medio per cápita familiar de las maricultoras pasó de $\mathrm{R} \$ 342,00$ para $\mathrm{R} \$ 488,80$ después del estudio, es decir, que representó un aumento del $29,9 \%$ sobre el ingreso familiar per cápita actual. Por lo tanto, los datos de esta investigación indican que la actividad económica de la venta de productos procedentes de algas G. birdiae desarrollado por la Asociación de Maricultoras de Río Fogo - AMAR tiene una gran importancia social y económica para las familias de ésta comunidad.

Palabras clave: maricultura; Gracilaria birdiae; sostenibilidad; comunidades costeras

\section{Introdução}

As algas são usadas para o consumo dos seres humanos há muitos anos, principalmente pelos chamados povos orientais, que possuem o costume de aproveitar uma ampla variedade dos recursos marinhos como alimento (Bezerra, 2008) devido ao elevado valor nutricional, sendo rica em proteínas, minerais, carboidratos e vitaminas. Além disso, o grande interesse econômico também é justificado pela crescente demanda por ficocolóides, para diferentes usos nas indústrias farmacêuticas, alimentícia e de cosméticos (Araujo and Rodrigues, 2011). 
Dentre os vários produtos extraídos de algas, o ágar é o que mais se destaca, devido a sua grande utilização e demanda no mercado mundial, figurando como um dos mais importantes dentre os ficocolóides (McHugh, 2003). O ágar é um polissacarídeo extraído de algumas famílias de Rhodophytas, principalmente das famílias Gelidiaceae e Gracilariaceae (Bezerra and Marinho-Soriano, 2010).

As comunidades costeiras Nordestinas estão entre as mais pobres e marginalizadas do Brasil, dependendo exclusivamente da pesca artesanal para o seu sustento. Nos últimos anos tem-se observado uma grande migração de populações residentes no interior para as comunidades costeiras em busca de novos meios de subsistência, fazendo com que haja conflitos sociais. Aliado a isto, a depleção dos estoques pesqueiros (peixes, crustáceos e mariscos) fez com que a comunidade procurasse novos bancos pesqueiros, incluindo as algas, que estão cada vez mais longe da costa (Bezerra and MarinhoSoriano, 2010).

Durante muito tempo as algas foram provenientes do extrativismo. A retirada descontrolada dos bancos naturais fez com que as populações de diversas espécies de algas diminuíssem principalmente as espécies de valor comercial. Assim, o cultivo de algas marinhas além de ser uma alternativa econômica, passou a ser também uma alternativa ecológica de uso dos recursos naturais (Muñoz et al., 2004).

Apesar dessa situação, fazem da pesca, no RN, a sua principal fonte de proteínas e renda aproximadamente 30 mil pescadores, sendo $30 \%$ deles mulheres (BRASIL, 2010). Dentre os segmentos pesqueiros, em área costeira, está o da maricultura que na região Nordeste é marcado pela presença feminina. O tema "gênero e pesca", pouco explorado no mundo acadêmico, consiste numa problemática instigante porque o mundo da pesca ainda é considerado um universo masculino, em que pese à participação das mulheres em diferentes atividades dessa cadeia produtiva (Maia and Neto, 2012).

A FAO, entre os anos de 2001 a 2003, implantou um projeto nos estados do Ceará, Rio Grande do Norte e Paraíba chamado "Desenvolvimento de comunidades costeiras" que visava verificar a viabilidade do cultivo de algas e outros produtos hidrobiológicos, a fim de expandir para outras áreas e comunidades. A Associação das Maricultoras de Rio do Fogo - AMAR é um fruto deste projeto, sendo um referencial para as demais comunidades que utilizam dessa prática (Bezerra and Marinho-Soriano, 2010). Assim como ocorre no estágio produtivo, a área financeira e de gestão também são alvos de pouco trato, haja vista as condições técnicas das marisqueiras, principalmente pelo baixo nível de escolaridade - entre elas, $17 \%$ são analfabetas (BRASIL, 2006). Logo o conhecimento das condições econômicas de qualquer atividade é fator de maior relevância para compreensão do que ocorre no setor, bem como para previsão de tendências futuros para a atividade. Desta forma, este estudo se posiciona como ferramenta para preencher esta lacuna, fornecendo dados econômicos que fundamentem e embasem a prática como uma atividade sustentável.

\section{Material e Métodos}

\section{Descrição da área}

O município de Rio do Fogo, localizado no estado do Rio Grande do Norte, limita-se ao Norte com o Oceano Atlântico e Touros; ao Sul com Maxaranguape; a Leste com o Oceano Atlântico e Maxaranguape; Oeste com Touros e Pureza, no litoral norte do estado (Figura 1). Possui uma área de $150 \mathrm{~km}^{2}$, população de 10.059 habitantes (IBGE, 2010) e $81 \mathrm{~km}$ de distância da capital. Apresenta um PIB per capita de R \$3.729,58, IDH 0,598 e esperança de vida ao nascer de 63,9 anos. O clima é tropical chuvoso, com temperatura média $25^{\circ} \mathrm{C}$, UR $75 \%$ e 2.700 horas de insolação.

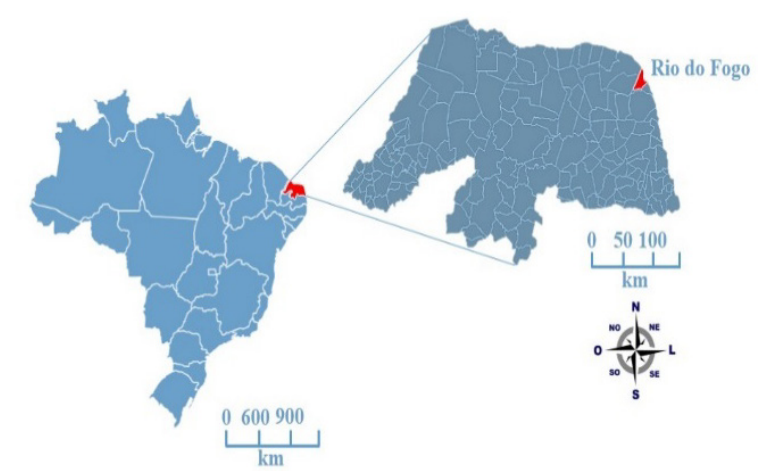

Figura 1. Localização do município de Rio do Fogo, Rio Grande do Norte.

Para coletar os dados, foram realizadas oficinas em Rio do Fogo e uma visita às áreas de cultivo, no ano de 2014. Foram aplicadas metodologias participativas entre os associados, com o sentido genérico de procedimentos que permitem aos atores sociais participarem de maneira ativa em espaços públicos. Como estratégia metodológica, utilizou-se o Diagnóstico Rural Participativo 
(DRP) e suas ferramentas próprias de mobilização do conhecimento, as quais privilegiam espaços coletivos para realização de uma pesquisa, pois não usa questionários, ao mesmo tempo em que assume como pressuposto básico que todo conhecimento é uma produção social e que, portanto, encontram-se num constante processo de elaboração, reformulação e validação. O uso dessa ferramenta metodológica permite reconstruir todos os passos processuais da atividade, sua participação no cotidiano da comunidade e da família, as relações sociais e econômicas, bem como a gestão democrática e a viabilidade econômica deste empreendimento popular, no passado e no presente (Maia and Neto, 2012).

\section{Estudo de viabilidade econômica}

No contexto da metodologia participativa foi realizado um estudo de viabilidade econômica (EVE) do principal produto produzido pela associação, utilizando conceitos contábeis preconizados por Carvalho et al. (2004), como produção mensal (PM), custo variável médio (CVM), custo fixos (CF), custo total de produção mensal $(\mathrm{CT}=\mathrm{CF}+\mathrm{CVM})$, preço de venda $(\mathrm{PV})$, comissão de venda $(\mathrm{CV})$, custo proporcional ao preço $(\mathrm{CPP}=[\mathrm{CV} * \mathrm{PV}]+[\mathrm{Ip} * \mathrm{PV}])$, preço líquido $(\mathrm{PL}=\mathrm{PV}-\mathrm{CPP})$, ponto de equilíbrio $(\mathrm{PE}=$ $\mathrm{CM} / \mathrm{PL} / \mathrm{PM})$, renda mensal líquida $(\mathrm{RML}=$ $\left.\mathrm{PM}^{*} \mathrm{PL}\right)$ e resultado mensal da produção $(\mathrm{RMP}=$ RML-CT).

\section{Resultados e Discussão}

A Associação de Maricultoras de Rio do Fogo (AMAR) é composta por seis associadas formam efetiva no desenvolvimento das atividades. Assim, todo lucro resultante das atividades desenvolvidas pela associação é dividido entre as 6 associadas.

O sistema de produção de algas utilizado pela associação é o de balsas com 20 metros de comprimento, onde as mudas das algas com peso médio de 50 gramas são dispostas ao longo da balsa. Durante o cultivo o único manejo utilizado é a limpeza das balsas pela retirada de outras espécies de algas e alguns crustáceos indesejados a cada 15 dias. Em 60 dias é realizada a retirada das algas, com produção de $1000 \mathrm{~kg} /$ balsa. Segundo Bezerra and Marinho-Soriano (2010) a utilização do sistema de balsas é o mais indicado para o cultivo de espécies marinhas. Logo após a retirada da balsa, as algas são lavadas e submetidas ao processo de secagem. Segundo Góes and Reis (2011) após o processo de secagem o rendimento da alga é de apenas $10 \%$, ou seja para $1.000 \mathrm{~kg}$ de alga úmida, apenas $100 \mathrm{~kg}$ é resultante deste processo. Desta produção, a associação produz sete produtos, são eles, a alga seca in natura, o pó de algas desidratado, sabonete de algas, gelatina de algas, cocada de algas e o mouse de algas. Todos estes produtos são comercializados na própria comunidade aos visitantes, apenas o pó de algas, é comercializado para indústrias de cosméticos.

Com a venda dos produtos a associação obtém renda mensal de $\mathrm{R} \$ 1.800,00 /$ mês, totalizando R\$ 300,00 associado/mês. Dentre os produtos comercializados na associação o pó de algas desidratado é o principal responsável pela lucratividade, representando $50 \%$ do total (Figura 2).

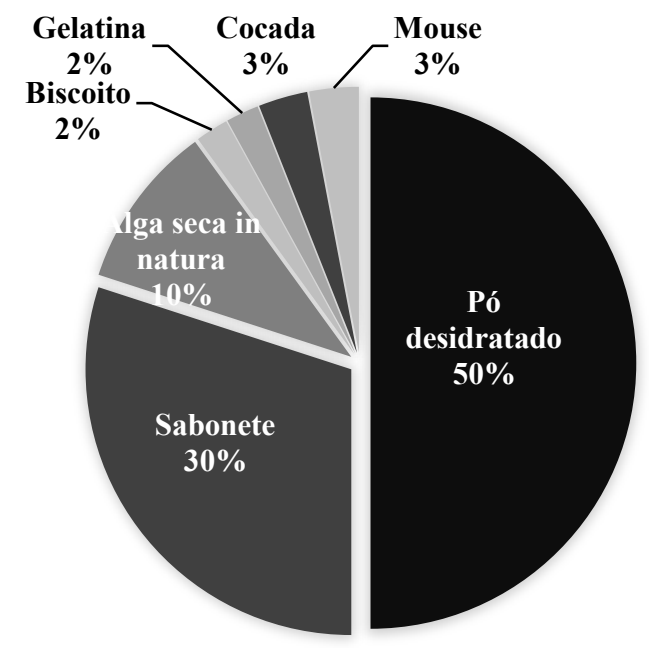

Figura 2. Lucratividade dos principais produtos comercializados na associação.

Para o estudo de viabilidade econômica foi utilizado o pó de algas desidratado. Segundo Góes and Reis (2011), o investimento necessário para implantação de um sistema de cultivo de balsas com capacidade de produção e processamento de $2.500 \mathrm{~kg} / \mathrm{mês}$ é de R $\$ 8.832,00$ (Tabela 1 ).

$\mathrm{O}$ custo fixo (CF) foi composto por produtos que independem da produção para tê-los, como a depreciação da estrutura e aluguel do triturador. O custo variável $(\mathrm{CV})$ foi composto pelo material necessário para desenvolver o produto, como embalagens, etiquetas, transporte, água e energia (Tabela 2). Como o estudo de viabilidade é sobre o pó de algas, no processo de moagem da alga seca se estabelece $10 \%$ de perca na trituração. Sendo assim, para $2.500 \mathrm{~kg}$ de alga úmida obtém $250 \mathrm{~kg}$ de alga seca, consequentemente $225 \mathrm{~kg}$ de pó. 
Dessa forma, mantém-se a meta de produção mensal (PM) de 450 pacotes de $500 \mathrm{~g}$.

Tabela 1. Investimentos necessários para implantação de um sistema de produção e beneficiamento de algas com capacidade de $2.500 \mathrm{~kg} / \mathrm{mês}$

\begin{tabular}{|c|c|c|c|c|}
\hline Itens & Quantidade & Unidade & $\begin{array}{c}\mathrm{R} \$ \\
\text { unitário } \\
\end{array}$ & $\begin{array}{l}\mathrm{R} \$ \\
\text { total }\end{array}$ \\
\hline Corda 10mm & 100 & $\mathrm{~m}$ & 0,0 & 170,00 \\
\hline $\begin{array}{l}\text { Cano } 5 \mathrm{~m} \text { cada } \\
75 \mathrm{~mm}\end{array}$ & 13 & $\mathrm{~m}$ & 0,0 & 611,00 \\
\hline $\begin{array}{l}\text { Rede revestimento } \\
\text { tubular }\end{array}$ & 400 & $\mathrm{~m}$ & 0,0 & 120,00 \\
\hline $\begin{array}{l}\text { Cordão seda } \\
6 \mathrm{~mm}\end{array}$ & 5 & $\mathrm{~kg}$ & 0,0 & 3,75 \\
\hline Poita & 2 & un. & 135,0 & 270,00 \\
\hline Lona $50 \mathrm{~m}^{2}$ & 1 & un. & 150,0 & 150,00 \\
\hline $\begin{array}{l}\text { Caixa d'agua } \\
5001\end{array}$ & 5 & un. & 104,9 & 524,50 \\
\hline Monobloco & 3 & un. & 13,5 & 40,50 \\
\hline Corda para poita & 24 & $\mathrm{~m}$ & 1,7 & 40,80 \\
\hline Saco de rafa & 10 & un. & 0,5 & 5,00 \\
\hline Ripa & 840 & $\mathrm{~m}$ & 0,9 & 756,00 \\
\hline Caibo & 560 & $\mathrm{~m}$ & 1,7 & 952,00 \\
\hline Prego para ripa & 10 & $\mathrm{~kg}$ & 5,5 & 55,00 \\
\hline Prego para caibo & 10 & $\mathrm{~kg}$ & 5,5 & 55,00 \\
\hline Tela sombrite & 40 & $\mathrm{~m}$ & 4,7 & 188,00 \\
\hline Balde plástico 6L & 18 & un. & 5,0 & 90,00 \\
\hline Carro de mão & 2 & un. & 105,0 & 210,00 \\
\hline $\begin{array}{l}\text { Máscara de } \\
\text { mergulho }\end{array}$ & 6 & un. & 75,6 & 453,60 \\
\hline $\begin{array}{l}\text { Boia de } \\
\text { sinalização }\end{array}$ & 6 & un. & 135,0 & 810,00 \\
\hline $\begin{array}{l}\text { Nadadeira (pé de } \\
\text { pato) }\end{array}$ & 6 & par & 90,0 & 540,00 \\
\hline $\begin{array}{l}\text { Sapatilha de } \\
\text { proteção }\end{array}$ & 12 & par & 50,0 & 600,00 \\
\hline Faca inox & 12 & un. & 6,0 & 72,00 \\
\hline Escova média & 12 & un. & 2,5 & 30,00 \\
\hline Martelo & 2 & un. & 16,3 & 32,54 \\
\hline Alicate & 2 & un. & 8,3 & 16,52 \\
\hline Arco de serra & 4 & un. & 11,0 & 44,00 \\
\hline Geladeira & 1 & un. & $1.000,0$ & 1.000 \\
\hline Fogão 4 bocas & 1 & un. & 500,0 & 500,00 \\
\hline Bolsa de cultivo & 1 & un. & 492,4 & 492,40 \\
\hline Total & & & & $8.832,6$ \\
\hline
\end{tabular}

A elaboração do estudo de viabilidade econômica (EVE) propicia o levantamento de informações importantes para a gestão e a sustentabilidade dos empreendimentos econômicos, inclusive os associativos (Costa, 2009). A busca dos números necessários para fazer as contas do EVE ajuda na construção de relações democráticas de trabalho, pois pode dar conta de: a) identificar os números do EVE e b) avaliar e debater a distribuição vigente das tarefas na perspectiva da construção da gestão democrática (Costa, 2009, Maia and Neto, 2012).

Tabela 2. Capital e custos de produção para produção mensal (PM) de 450 pacotes de pó de algas.

\begin{tabular}{|c|c|c|}
\hline \multicolumn{3}{|c|}{ Capital empatado } \\
\hline Discriminação & Valor $(\mathrm{R} \$)$ & Participação (\%) \\
\hline Valor total do investimento & $8.832,00$ & 100,00 \\
\hline \multicolumn{3}{|c|}{ Custos fixos } \\
\hline Depreciação & 24,34 & 3,14 \\
\hline Aluguel de triturador & 750,00 & 96,86 \\
\hline$\underline{\text { Subtotal }}$ & 774,34 & 100,00 \\
\hline \multicolumn{3}{|c|}{ Custos variáveis } \\
\hline Embalagens & 135,00 & 26,73 \\
\hline Etiquetas & 135,00 & 26,73 \\
\hline Transporte & 120,00 & 23,76 \\
\hline Água & 25,00 & 4,95 \\
\hline Energia & 90,00 & 17,82 \\
\hline Subtotal & 505,00 & 100,00 \\
\hline Custo Total & \multicolumn{2}{|c|}{$1.279,00$} \\
\hline
\end{tabular}

Como estimativas dos parâmetros do estudo de viabilidade econômica foram registrados custo variável médio (CVM), obtido pela divisão do custo variável mensal pelo nível de produção mensal, resultando em um CVM de R $\$ 1,12$. O custo proporcional ao preço (CPP), obtido através do produto entre o preço de venda $(\mathrm{R} \$ 10,00)$ e a comissão de vendas (4\%), mais o produto entre o preço das vendas (PV) e os impostos pagos (17\%) que foram estabelecidos pelas integrantes do grupo em, resultando em um CPP de R $\$ 2,10$.

O preço líquido foi determinado através da diferença entre o preço de venda e o custo proporcional ao preço, ficando em $\mathrm{R} \$$ $7,90 /$ unidade de $500 \mathrm{~g}$. O ponto de equilíbrio (PE) foi de $35,6 \%$, resultado da razão do custo total com o preço líquido e a quantidade vendida. Segundo Bornia (2010) o ponto de equilíbrio é o nível de vendas onde o lucro é nulo. É um dos indicadores que informa sobre o volume necessário de vendas em um determinado período para cobrir todos os custos fixos e variáveis, quanto menor o PE maior sua lucratividade.

A receita mensal líquida (RML) foi obtida através da razão entre a quantidade vendida e o preço líquido, resultando em uma RML de R\$ $3.555,00$. O resultado mensal da produção (RMP) foi R $\$ 2.276,00$. Após a divisão da RMP entre os associados, cada um receberá ao final de cada mês $\mathrm{R} \$ 379,33$, valor este maior que o encontrado por 
Maia and Neto (2012) de R\$ 153,76 para marisqueiras de uma comunidade do semiárido do mesmo estado, esta diferença pode ser explicada pelo fato de que a forma de comercialização é diferente, onde produtos processados agregam maior valor do que os comercializados de formas tradicionais.

Além dos trabalhos desenvolvidos na associação, as associadas realizam atividades extras para incremento da renda, como ambulantes e auxiliar de serviços gerais. A renda oriunda das atividades extras não ultrapassa R \$300,00/mês. A figura 3 nos mostra quão importante é o investimento na associação, pois a média da renda dessas mulheres, sem os lucros da venda do pó não ultrapassa $\mathrm{R} \$ 150,00 /$ mês. Como relatado anteriormente o pó desidratado é responsável por $50 \%$ de toda lucratividade da associação, por isso não é somado aos lucros dos outros produtos para enfatizar a renda do pó após o investimento.

Após o investimento, as associadas passaram a ter renda próxima de um salário mínimo (R\$ $880,00)$. Segundo Vasconcelos et al. (2003) 70\% dos pescadores artesanais e pequenos maricultores possuem renda mensal de até um salário mínimo, mesmo patamar encontrado nas maricultoras de Rio do Fogo.

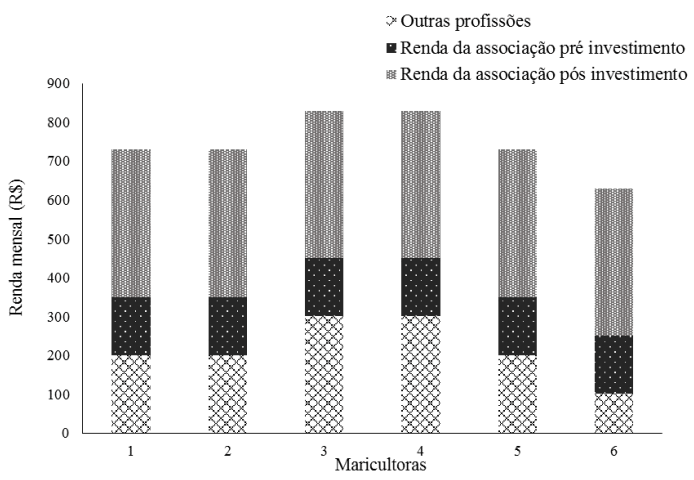

Figura 2. Renda da mensal das maricultoras.

Segundo o IBGE (2010) a renda familiar per capita do município de Rio do Fogo é de R\$ 236,00/mês. Quando comparado com a renda atual das famílias da maricultoras que é de $\mathrm{R} \$$ $342,00 /$ mês, estão acima $42,6 \%$ acima da média encontrada pelo senso no ano de 2010. Após o investimento a renda das famílias passaria a ser $\mathrm{R} \$$ 488,80 , ou seja, $100 \%$ acima da renda do município e 29,8\% acima da renda das maricultoras. Assim, contribuindo para redução da desigualdade.
A renda mensal da associação é expressa na Figura 4. Onde após o investimento é possível ter um acréscimo de $176 \%$ na renda mensal.

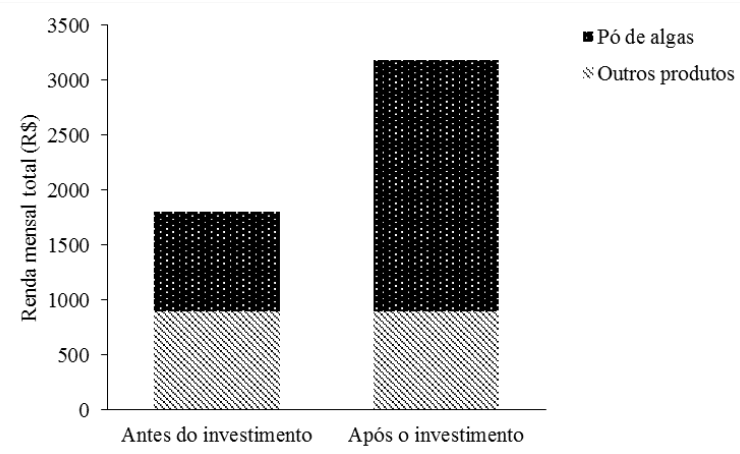

Figura 4. Renda mensal da associação antes do investimento e após o investimento.

\section{Conclusões}

Associação de Maricultoras de Rio do Fogo AMAR tem uma grande importância social e econômica, tendo em vista o que a própria associação conquistou e tem conquistado ao longo desses 10 anos de trabalho e as famílias que tem aumentado a sua renda através dessa atividade. $\mathrm{O}$ estudo de viabilidade econômica demonstrou que o investimento visando uma maior produção de algas é economicamente viável, trazendo lucros para a associação, aumentando a rendas das famílias das maricultoras consideravelmente e consequentemente dando uma maior qualidade de vida para às associadas que tanto se dedicam à atividade e agora têm suas esperanças renovadas com o resultado deste estudo.

\section{Referências Bibliográficas}

Araujo, G. S. \& Rodrigues, J. A. G. 2011. Maricultura da alga marinha vermelha Gracilaria birdiae em Icapuí, Ceará. Arquivos de Ciência do Mar, 44, 62-68.

Bezerra, A. F. 2008. Cultivo de algas marinhas como desenvolvimento de comunidades costeiras. Animal Science. Universidade Federal do Rio Grande do Norte, João Pessoa.

Bezerra, A. F. \& Marinho-Soriano, E. 2010. Cultivation of the red seaweed Gracilaria birdiae (Gracilariales, Rhodophyta) in tropical waters of northeast Brazil. Biomass and Bioenergy, 34, 1813-1817.

Bornia, A. C. 2010. Análise gerencial de custos: aplicação em empresas modernas. Atlas, São Paulo. 
BRASIL. 2006. Resultados do recadastramento nacional dos pescadores do Brasil. Brasília, DF: SEAP/PR.

Carvalho, R. C. A., Chaves, R. A. \& Cintra, I. H. A. 2004. Análise de custos e rentabilidade de embarcações industriais envolvidas na captura de Piramutaba, Brachyplatystoma Vaillantii Valenciennes, 1940), no estuário do Rio Amazonas, litoral Norte do Brasil. Boletim Técnico Científico do CEPNOR, 4, 45-56.

Costa, R. 2009. Estudo de viabilidade econômica: uma perspectiva de apropriação pelos empreendimentos associativos. In: Lobato, R. \& Fonseca, M. (eds.) Uma proposta de formação: desmistificando os números. Catarse, Porto Alegre.

Góes, H. G. \& Reis, R. P. 2011. An initial comparison of tubular netting versus tie-tie methods of cultivation for Kappaphycus alvarezii (Rhodophyta, Solieriaceae) on the south coast of Rio de Janeiro State, Brazil. Journal of Applied Phycology, 23, 607-613.

IBGE - Instituto Brasileiro De Geografia E Estatística (2010). Indicadores de desenvolvimento sustentável. Disponível em: $<$ http://www.ibge.gov.br/home/geociências/re cursosnaturais/ids/default.shtm $>$. Acesso em: 23 de Novembro de 2016.

Maia, I. S. \& Neto, J. T. O. 2012. Estudo de viabilidade econômica e gestão democrática de empreendimentos populares: o caso das marisqueiras do semiárido potiguar. Vivência: Revista de Antropologia, 40, 67-79.

McHugh, D. J. 2003. A guide to the seaweed industry. Food and Agriculture Organization of the United Nations Rome, Rome.

Muñoz, J., Freile-Pelegrín, Y. \& Robledo, D. 2004. Mariculture of Kappaphycus alvarezii (Rhodophyta, Solieriaceae) color strains in tropical waters of Yucatán, México. Aquaculture, 239, 161-177.

Vasconcelos, E. M. A., Lins, J. E., Matos, J. A., Junior, W. \& Tavares, M. M. 2003. Perfil socio-economico dos produtores da pesca artesanal maritima do Estado do Rio Grande do Norte. Boletim Técnico Cientifico do CEPENE, 11, 277-292.

\section{Article History:}

Received 23 December 2016

Accepted 18 January 2017

Available on line 26 January 2017

License information: This is an open-access article distributed under the terms of the Creative Commons Attribution License 4.0, which permits unrestricted use, distribution, and reproduction in any medium, provided the original work is properly cited. 